\title{
Los migrantes coreanos en la industria textil de la Ciudad de Buenos Aires. Inserción económica e identidades urbanas
}

Les migrants coréens dans l'industrie textile de la ville de Buenos Aires.

Intégration économique et identités urbaines

Korean Migrants in the Buenos Aires Garment Industry. Economic Integration and Urban Identities

\section{Carolina Mera}

\section{OpenEdition}

\section{Journals}

Edición electrónica

URL: https://journals.openedition.org/remi/6221

DOI: 10.4000/remi.6221

ISSN: $1777-5418$

Editor

Université de Poitiers

Edición impresa

Fecha de publicación: 1 diciembre 2012

Paginación: 67-87

ISBN: 979-10-90426-06-1

ISSN: 0765-0752

\section{Referencia electrónica}

Carolina Mera, «Los migrantes coreanos en la industria textil de la Ciudad de Buenos Aires. Inserción económica e identidades urbanas», Revue européenne des migrations internationales [En línea], vol. 28 n4 | 2012, Publicado el 01 diciembre 2015, consultado el 15 abril 2022. URL: http://

journals.openedition.org/remi/6221 ; DOI: https://doi.org/10.4000/remi.6221 


\section{Los migrantes coreanos en la industria textil de la Ciudad de Buenos Aires. Inserción económica e identidades urbanas}

\section{Carolina Mera ${ }^{1}$}

\section{Introducción}

El estudio del caso coreano en la industria textil es relevante porque presenta patrones de inserción y movilidad muy interesantes en función del tipo de proceso de instalación urbana, donde la red étnica y las redes transnacionales condicionan la manera de incorporarse en el mundo económico. Además, en este tipo de instalación económica prima la tendencia a generar actividades de micro-emprendimientos independientes, pudiendo hablarse de empresariado migrante. ${ }^{2}$ Siguiendo esta línea, adquiere relevancia el hecho de que la mayor parte de la actividad económica de la comunidad coreana en Argentina se concentra en la pequeña y mediana industria textil y en el comercio de indumentaria, mayorista y minorista.

En general, a pesar del proceso de recesión económica que vivió la Argentina en las últimas décadas, los miembros de esta comunidad han protagonizado un acelerado proceso de movilidad social ascendente. ${ }^{3}$ Este vertiginoso mejoramiento de su situación económica está relacionado, como se verá a lo largo del trabajo, por un lado, con el tipo de organización característica del rubro textil, y por otro, con la propia organización comunitaria, basada en la familia

1 Investigadora CONICET, Profesora Universidad de Buenos Aires, Consejo Nacional de Investigaciones Científicas y Técnicas, av. Rivadavia 1917 (C1033AAJ) Ciudad Autónoma de Buenos Aires, República Argentina; carolinamera@yahoo.com

2 El artículo es parte de una larga investigación sobre las comunidades coreanas en Argentina y América Latina. Se parte de una perspectiva de trabajo cualitativa interdisciplinaria que recurre a datos secundarios y a material de trabajo original relevado en las diferentes ciudades estudiadas. En este caso nos centraremos en los relevados en la Ciudad de Buenos Aires desde la instalación de la comunidad hasta el 2012.

3 El relativo éxito económico se realizó a costa de un gran sacrificio físico, afectivo y psicológico de todos los miembros de la familia. Este éxito debe medirse en función del nivel de preparación que traen de Corea y del esfuerzo y la entrega que ponen en el trabajo (el tiempo, la dedicación y el número de familiares que trabajan). Es decir, lograron una mejoría económica en los inicios que luego se vio fortalecida en la interacción con otros grupos migrantes como bolivianos que recién entraban en el sistema de producción textil. 
y dentro de la cual prima una fuerte solidaridad grupal. Estos procesos pueden ser explicados desde el concepto de empresariado migrante y enclave étnico. Se analizan en el presente texto los comportamientos en el área económica del rubro textil que adquieren una dimensión significativa a partir de las lógicas transnacionales y del tipo de instalación local caracterizada por la concentración social y espacial.

Dada la estrecha relación entre la concentración espacial urbana y el tipo de organización de la actividad económica, se enmarca el estudio a partir del concepto de enclave étnico, como lo entienden Wilson y Portes (1980: 295-319) en el sentido de un grupo de inmigrantes que se concentra en un espacio distintivo y organiza una serie de empresas que sirven para su propia comunidad étnica y/o para la población en general, espacio que posibilita alcanzar niveles importantes de auto-empleo a partir de la movilización de recursos étnicos materiales y simbólicos. Si bien se considera que muchas de las dimensiones propuestas por esos autores están entreveradas en el caso que a la investigación concierne, se decidió también incorporar la utilización del concepto de empresariado étnico, entendido en el sentido complejo que le adjudican Waldinger, Aldrich y Ward (1990), como el conjunto de actividades empresariales realizadas por personas pertenecientes a un grupo de origen migrante con una significativa dependencia del capital social proporcionado por los recursos étnicos, de una manera adaptada a las condiciones en destino. Se complementan los ejes citados con los aportes de Min y Kim (1999) quienes incorporan al análisis la noción de identidad étnica como la clave de la adhesión y de la solidaridad entre los miembros del grupo, refiriéndose, por un lado, a los elementos relacionados con la situación previa a la migración que garantizarían cohesión, y por otra parte, a la dimensión en que esta identidad es recreada de forma dinámica en el contexto de la sociedad receptora.

La concentración de los migrantes coreanos en pequeños emprendimientos productivos y comerciales puede ser explicada a partir de argumentos culturales - que priorizan aspectos como la confianza, la ayuda mutua, la capacidad para articular actividades conjuntas y para utilizar sistemas de crédito rotativo y otros comportamientos típicos del país de origen (Light, 1972; Yoon, 1991: 303-332) - y conjuntamente a través de otras perspectivas, que la abordan desde el rol de "middleman minorities» (Bonacich, 1973: 583-594; Min, 1990: 436-455). Esta última supone que los grupos de migrantes privilegian el compromiso con la sociedad de origen a la que esperan volver. Esto justificaría la concentración en actividades étnicas y los ubicaría en la mediación entre ciertos sectores dominantes, elite de la sociedad global y ciertos grupos subalternos, como otros migrantes. En este sentido, el tipo de actividad e inserción económica agrupa variables étnico-culturales, pero también otras como la formación educativa y social general de los migrantes, las experiencias laborales previas a la migración, y los conocimientos y competencias que les permiten establecerse como emprendedores independientes, además de la voluntad de llevar adelante estos procesos y el acompañamiento familiar (Hurh, 1980: 1-36; Kim y Hurh, 1984: 696-713). Como sostienen Waldinger, Aldrich y Ward (1990), el contexto de recepción produce una concentración espacial que desemboca en la formación del enclave económico, que a la vez se vuelve un espacio donde estas personas migrantes reproducen tanto el sentido de comunidad y de adhesión identitaria al grupo como una fuerte solidaridad étnica. 
Para dar cuenta de la articulación entre la forma de instalación, la importancia de la red y del capital social étnico y la actividad económica en la conformación de estos emprendedores étnicos, se exponen, en primer lugar y brevemente, las características generales del proceso de instalación. Luego se introducen aspectos sobre la importancia del capital social en estos procesos. En tercer lugar se hará una breve caracterización de la comunidad coreana como organización étnica y su concentración urbana seguida de una síntesis sobre la situación de la industria textil en Argentina en las últimas décadas. En este punto se da cuenta de las etapas y características de la instalación de estos migrantes en la industria textil y su conformación como empresariado étnico. Finalmente se delinean algunas reflexiones.

\section{Característica general del proceso de instalación. Un poco de historia}

Los primeros inmigrantes coreanos que llegaron a Argentina lo hicieron por tierra, desde Bolivia y Paraguay. De esta manera, cuando en 1965 desembarca el primer barco oficial de inmigrantes coreanos, ya había muchas familias coreanas viviendo en el país. Hasta principios de 1970 llegan flujos aislados de migrantes y al finalizar esa década desembarca un centenar de familias para establecerse en áreas rurales. Sin embargo, los asentamientos rurales no prosperan y estas familias terminan instalándose en las grandes ciudades.

En Abril de 1984 se firma el Acta de Procedimiento para el ingreso de inmigrantes coreanos a la Argentina, ${ }^{4}$ por la que se otorgan más de 11.000 permisos de ingresos familiares. Este nuevo flujo migratorio se diferencia de los anteriores porque trae capital para invertir y migra de una Corea moderna e industrializada. A partir de ese momento se puede señalar la consolidación de la comunidad coreana en Buenos Aires. En trabajos anteriores se han delineado dos momentos en el proceso de instalación: uno, que podría llamarse la "época de oro de la migración coreana en Argentina" - desde fines de los años 1980 hasta principios de los años 1990 -, momento en el que se consolida la organización institucional; y otro, que acompaña la agudización de la crisis económica que vivió el país a partir del año 2000, caracterizado por la constante disminución cuantitativa y pérdida de espacios de sociabilidad de la comunidad (Mera, 2008: 1-10). Las décadas de 1990 y 2000 no mostraron entrada de nuevos migrantes sino re-emigración. ${ }^{5}$ Actualmente se estima en alrededor 23.000 el número de residentes coreanos en Argentina, como se puede observar en el siguiente gráfico.

4 Ver Martin Saravia, 1988: 179-189.

5 El fenómeno de la re-emigración de los migrantes coreanos en América Latina refiere principalmente a los desplazamientos intra-regionales que se producen una vez instalados en la región. Si bien las familias se instalan en un primer país, luego se produce lo que hemos Ilamado movimientos de re-emigración (Mera, 2008: 1-10; 2010: 1-18). 
Figura 1: Los residentes coreanos en Argentina

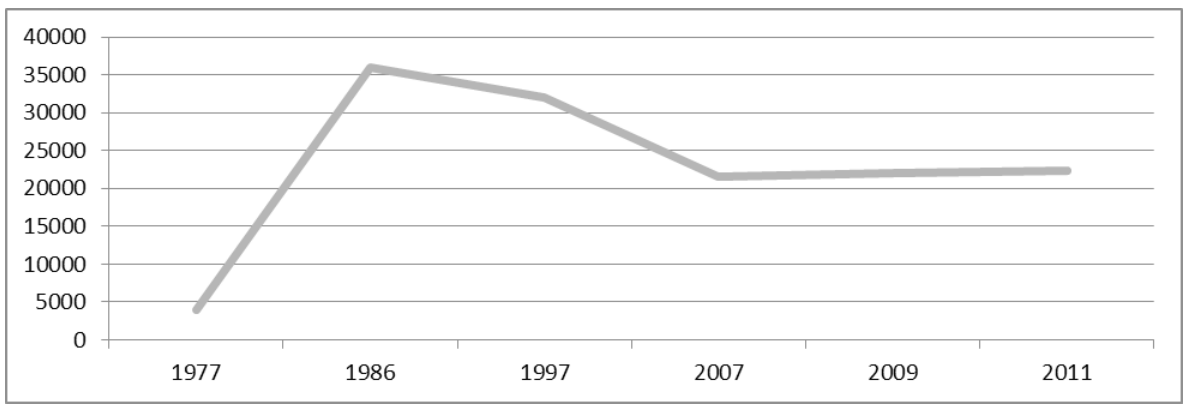

Fuente: Elaboración propia con datos obtenidos de las Instituciones de la Comunidad coreana de Argentina, de Lee (1992), de la Dirección Nacional de Migraciones del Ministerio del Interior y Transporte y del Departamento de Migraciones del Ministerio de Relaciones

Exteriores y Comercio de Corea (2005-2011).

La llegada a Buenos Aires. Si bien los primeros emprendimientos se dirigían hacia colonias rurales, éstos no prosperaron por las condiciones desfavorables de las tierras para el cultivo, por la falta de infraestructura básica en las cercanías de los lugares y también porque mucha de la población no provenía de zonas de cultivos ni tenía experiencia rural. Por estas razones, la mayor parte de las familias se trasladó a las ciudades para poder prosperar en su economía y calidad de vida.

En la Ciudad de Buenos Aires desde muy temprano adoptaron una fuerte tendencia a concentrase en ciertos barrios. Primeramente se establecieron en villas de emergencia, ${ }^{6}$ para luego asentarse en barrios como Soldati, Villegas, el Complejo departamental de Ciudadela y Presidente Mitre, en el Bajo Flores. De esta época data el nombre del Barrio Rivadavia, que reúne la principal concentración de coreanos en la Ciudad de Buenos Aires hasta la actualidad: Baek-ku (109). ${ }^{7}$ Con el tiempo irán concentrándose en pocos barrios, que coinciden con el lugar de desarrollo de las actividades de producción textil.

Sin duda la formación del barrio coreano puede ser entendida como un enclave étnico, en el sentido de reflejar la expresión espacial de la concentración de población inmigrante, y la economía étnica que, como se puede apreciar, se fortalece gracias a la consolidación de las redes sociales que los recursos comunitarios movilizan.

6 La llegada a estos barrios precarios significó un descenso en el status que tenían en su país, ya que provenían de los sectores medios de la sociedad. Entre ellos se contaban médicos, profesores universitarios y secundarios, técnicos de televisión, varios religiosos, empleados públicos y administrativos.

7 Número del bus que unía la Estación de Retiro con el Bajo Flores. 


\section{La importancia del capital social de la diáspora}

Se considera que el concepto de diáspora y especialmente su dimensión transnacional es fundamental para explicar el funcionamiento de la economía étnica en la medida en que se constituye en capital social con un fuerte impacto en la vida económica de los migrantes (Mera, 2011: 201-212). El transnacionalismo diaspórico garantiza redes familiares, comunicacionales, religiosas, culturales, económicas y financieras que permiten a los migrantes mejores condiciones de desplazamiento. ${ }^{8}$

Este análisis entiende que la diáspora pone de relieve el territorio como entidad física y simbólica, constituida por tres ejes: los anclajes nacionales, la identidad en el desplazamiento y la conciencia nacional de origen. Es decir, la identidad no se transfiere mecánicamente de Corea sino que es reelaborada sobre la base de interacciones entre estructuras sociales, contradicciones de clase y modelos culturales tanto del país de origen como del de recepción. La diáspora es tomada como una usina de identidad distintiva, producto de procesos de hibridez, fluidez y cambio, que dan cuenta de la complejidad de las relaciones que intervienen a lo largo de la construcción identitaria (Mera, 2010: 1-18). La articulación de los procesos estructurales-macrosociales y los subjetivos-microsociales, pone en tensión las identidades y las subjetividades en juego. Por eso se recurre al Paradigma de la movilidad al que refiere Tarrius (2000: 37-66) donde la idea de "territorio circulatorio" permite aprehender la socialización de espacios según lógicas de movilidad. El par movilidad/alteridad reemplaza en el esquema de las relaciones transnacionales a los conceptos de integración-identidad del pensamiento nacional. La migración será sólo un nivel de ese proceso mayor que reúne la tríada identidad-espacio-tiempo, de sujetos de "aquí» y "allá», de micro espacios urbanos y redes macro de circulación transnacional.

Un elemento importante para trabajar sobre las comunidades coreanas, es que este concepto de diáspora permite dar cuenta de los procesos parciales de incorporación a las sociedades receptoras, y echar luz a las diferentes esferas sociales y a sus relaciones. Los miembros de una diáspora pueden estar integrados a la sociedad de recepción, pero esto no significa que estén asimilados, como en el caso que se está trabajando.

\section{Caracterización de la comunidad coreana como organización étnica}

El tipo de actividad económica está directamente relacionado con la forma de instalación en el espacio urbano y las formas de sociabilidad que de allí se desprenden, muy asociadas, como ya se mencionó, a su naturaleza diaspórica. En este sentido adquiere relevancia la relación actividad económica-ubicación

8 En este sentido es interesante el aporte de la reflexión acerca del contenido político y social que adquiere la posibilidad de movilidad espacial en la etapa actual del capitalismo (Bauman, 1999; Sassen, 1999). 
espacial en la Ciudad de Buenos Aires. ${ }^{9}$

La posibilidad de pensar el territorio ${ }^{10}$ desde su dimensión cultural (Sassone, 2002) permite acceder a ciertas lógicas que conforman la base de las relaciones sociales, consolidadas por las cadenas y las redes migratorias. Los migrantes impactan en el espacio urbano en el que se asientan, estableciendo nuevas jerarquías sociales y redefiniendo su espacio social de pertenencia desde las nuevas territorialidades. De este modo, las jerarquías sociales establecidas en el seno de la cadena se extienden sobre la microcomunidad de radicación ya que un barrio étnico no es sólo el lugar físico donde se establecen los migrantes de un cierto origen, sino aquel espacio social donde siguen reproduciéndose las relaciones de poder y los sentidos sociales. Algunos autores sostienen que el agrupamiento de migrantes o de grupos étnicos cumple funciones de contención afectiva y protección en un entorno diferente, a partir de la conservación de costumbres y tradiciones y aumentando la cohesión (Kim y Hurh, 1993: 696-713; Min y Kim, 1999; Mera, 1998; Sassone, 2004: 177-197). Así, ese espacio de instalación deviene en barrio de migrantes o barrio étnico, en el que las personas desarrollan un sentido de pertenencia. Pero además, esos barrios apropiados condicionan, de manera particular, el proceso de inserción económica de los grupos migrantes. Por ejemplo, iglesias y asociaciones comunitarias son las que en general garantizan la circulación de información en relación a las posibilidades de trabajo, referencias y datos necesarios para la gestión de la economía étnica, familiar y grupal. ${ }^{11}$ Son fundamentales en la provisión de empleo y beneficios al grupo frente a la sociedad receptora. Además, se pudo constatar una preferencia a tratar con proveedores coreanos para puestos claves como las cajas, controles, contadores, abogados; y con empleados de otras nacionalidades para otras funciones: preferentemente bolivianos para los trabajos manuales en los talleres, y empleadas argentinas para la venta al público (Mera, 1998).

Es en este punto donde los procesos de construcción étnica se vuelven fundamentales para comprender cierto tipo de inserción y organización económica. Cuanto mayor es la adhesión de las personas al capital cultural, social y psicológico del grupo étnico, mayor será la forma en que las acciones étnicas colectivas se movilicen para mantener los intereses del grupo. En este sentido, como sostiene Min y Kim (1999), la identidad étnica es la clave de la adhesión y de la solidaridad intra-etnia. Según estos autores, las teorías de la etnicidad podrían ser clasificadas según dos perspectivas: por un lado la de la aproxima-

9 En las grandes ciudades, cuando los migrantes se concentran en barrios, se observan marcas culturales de cohesión étnica. Ciudades como Ámsterdam, Madrid, Toronto,

San Pablo, Nueva York, San Francisco, Los Ángeles, Roma, Milán y Londres, entre otras, cuentan con barrios de migrantes y centros comerciales étnicos. Incluso hay espacios públicos donde determinados días se reúnen los migrantes. Buenos Aires no es ajena a este fenómeno y presenta barrios de migrantes, como el Barrio Charrua (de bolivianos) y el barrio chino de Belgrano.

10 El concepto de territorio reúne las nociones de espacio de vida, de espacio social y de espacio vivido (Di Meo, 1991; Soja, 1997; Sassone, 2002, Massey, 2005; Arfuch, 2005).

$11 \mathrm{El}$ componente étnico en los centros urbanos ha sido desarrollado por distintas teorías: la escuela de Chicago de comienzos del siglo XX, en la que se destacaron Halbwachs, Park, Burguess y Mackenzie, estudiando la diferenciación social del espacio y los migrantes (Grafmeyer y Joseph, 1990); el enfoque sociológico marginalista que se basa en el estudio de los grupos sociales delimitados en un área ecológicamente marginal como "slum» (Mármora, 1971) y enfoques como el de Barth (1976) que plantea la noción de "límite étnico», definido según la existencia de "miembros" y "extraños". 
ción primaria, "primordial approach», que se refiere a los elementos relacionados con la situación previa a la migración: lengua, religión, rasgos físicos y lazos asociativos, entre otros. De acuerdo con esta perspectiva, un grupo étnico donde sus miembros comparten estas características, mantienen un alto grado de cohesión. Por otra parte, la "mobilizationist perspective» sostiene que la etnicidad es creada y recreada en el contexto de la sociedad receptora. Enfatizan la segregación, la concentración laboral, la discriminación y otros factores estructurales de la nueva sociedad. Para los autores la primera perspectiva daría cuenta de la cohesión y de los grados de adhesión étnica, mientras que la segunda lo haría sobre la solidaridad étnica (Min et Kim, 1999: 17).

Para el caso de los coreanos en la industria textil de Buenos Aires, estas perspectivas adquieren gran relevancia ya que nos permiten explicar las diferentes dimensiones del fenómeno. Desde la perspectiva del "primordial approach» abordamos los lazos primarios, afectivos, las emociones y las experiencias subjetivas que permiten crear la solidaridad para establecer los intercambios económicos. La continuidad de componentes étnicos como la lengua, la comida o la religión, mantienen en el tiempo la existencia del grupo, pero siempre y en todos los casos, la cohesión está atada al hecho de compartir la misma experiencia histórica. Por su parte, la "mobilizationist perspective» sostiene que mantener y desarrollar los lazos primarios depende de los factores estructurales de la sociedad receptora, en este sentido, la inserción urbana y la naturaleza productiva del área de la industria textil determinará la formación de enclaves étnicos.

Según Kim, Kim y Hurh (1981: 219-232) se pueden tomar varios indicadores que nos hablan del tipo de organización económica de los migrantes de acuerdo a las formas de conseguir empleo, que iría desde métodos de adquisición de información sobre empleo formal (medios masivos, centros públicos y privados, universidades, centros profesionales, etc., caracterizados por el uso de canales abiertos de transmisión e información sobre empleos) o de tipo más informal, relacionado al capital social de la comunidad, contactos personales que se dan como una búsqueda directa. El ámbito donde se desarrollan laboralmente, de más cerca a más lejos de la familia, amigos y familiares hasta empresas y espacios no conocidos. Otro eje es si trabajan para personas del grupo o en actividades que tienden a cubrir necesidades más amplias. En Argentina la primera generación obtiene empleo e información referida a trabajos a través de métodos informales. Esta situación reproduce la concentración étnica ya que se hace más difícil penetrar en otros círculos debido a la falta de información o de contactos. Sin embargo, debemos resaltar una notable diferencia entre la población profesional universitaria escolarizada. La mayoría de los contadores, abogados y economistas que trabajan para empresas (en relación de dependencia) encontraron sus puestos mediante entrevistas obtenidas de anuncios en los diarios.

La tendencia a la concentración no aparece como una etapa de instalación, sino que se estructura como el eje de la vida comunitaria, y especialmente de la vida económica. Esto explica la concentración espacial de las actividades económicas en Once y Avellaneda, y los servicios «étnicos» en Baek-ku, el Bajo Flores. 


\section{Situación de la industria textil en Argentina en las últimas décadas y su forma de organización social del trabajo}

El proceso de desindustrialización que se inicia en Argentina con el golpe militar de 1978 fue el punto de fuga de un largo proceso que dará al sector textil una particularidad. Las relaciones laborales tomaron características que se fueron agudizando en la década del 1990 debido a la inminente crisis. Las políticas de libre mercado y de promoción industrial mediante exenciones impositivas, promovieron procesos de relocalización territorial de la producción industrial en función de la obtención de menores costos de producción. Además, el tipo de cambio que establecía la paridad un peso argentino igual un dólar estadounidense, desfavorecía la exportación de las industrias nacionales y promovía las importaciones. Se vivió una crisis de grandes proporciones que llevó al cierre de establecimientos industriales y altas tasas de desempleo. El sector textil se caracterizó por "una tendencia global hacia la reorganización de la producción a través de diversas formas de subcontratación para que ésta sea cada vez más flexibilizada y descentralizada. Las grandes empresas contratan a proveedores, quienes, a su vez, distribuyen trabajo a subcontratistas, muchos de los cuales operan en la economía informal» (Benencia, 2009). Las diversas modalidades de "tercerización de la producción y el trabajo" involucran a trabajadores sujetos a la lógica de flexibilización laboral y a las coyunturas de los ciclos económicos, en general migrantes latinoamericanos indocumentados. ${ }^{12}$

En la actualidad, la industria textil y de la confección se encuentra en una etapa de estabilización, luego del leve crecimiento que se produjo entre el 2002 y el 2003. Sin embargo, aún existen talleres clandestinos, ubicados fundamentalmente en la Ciudad y en la provincia de Buenos Aires, donde las condiciones de trabajo siguen siendo muy precarias. "La cadena de valor de la industria de indumentaria es compleja y de difícil fiscalización. La mayoría de la producción se encuentra tercerizada fuera de las empresas, generalmente en más de un taller. La masa crítica de talleres efectivos donde las empresas-marcas tercerizan su producción es incalculable, en parte, debido a la informalidad característica del sector y su atomización, y al terror impuesto por la coerción de los dueños de talleres clandestinos donde existe trabajo esclavo" (D'Ovidio, 2007). Un hecho significativo es que, luego de la crisis económica que sufrió Argentina (2001-2002), muchos migrantes latinoamericanos se reacomodaron en la débil economía local, siendo la industria textil, de confecciones y del calzado uno de los rubros que más los albergó.

12 Los migrantes en la Ciudad de Buenos Aires. Según los datos del Censo de 2010, la población total de Argentina es de 40.117.096 habitantes. La población extranjera alcanza 1.805.957, un 4,5\% de la población total. El 3,5\% de esta población proviene de países limítrofes y del Perú (1.402.568 habitantes) mientras que el 0,7\% de Europa (299.394 habitantes). La Ciudad y la Provincia de Buenos Aires (CABA y PBA, respectivamente) albergan al $46 \%$ de la población total del país, y para el caso de los migrantes internacionales alberga al 73,3\%. Es decir, que el comportamiento de asentamiento de los migrantes repite y profundiza el del modelo de concentración poblacional que impera en el país y que manifiesta cada vez más patrones urbanos. Si se recorta sólo la migración proveniente de los países limítrofes y del Perú, dicha concentración es del 71,68\%, siendo casi el $86 \%$ de paraguayos, el $66 \%$ de bolivianos y el $82,5 \%$ de peruanos. En lo que refiere a las áreas económico-laborales, estos migrantes se incorporan a sectores de baja productividad como la construcción, el servicio doméstico, trabajadores fabriles con baja calificación (textil, confección y calzado), en sectores del mercado laboral con condiciones de trabajo más peligrosas y vulnerables. 
En el caso de los coreanos se ha observado cómo se han ido adaptando a los vaivenes del sector de acuerdo a su capacidad de acumulación de capital y a su mejor inserción en el país. Los coreanos participan en la cadena de empleadores, contratistas y subcontratistas que termina en formas de "trabajo a destajo", falta de condiciones adecuadas de trabajo, y otras pautas fuera de lo que establece la legislación laboral. En muchos casos los talleres están fuera de toda habilitación o marco regulatorio (tanto respecto de las relaciones laborales como de condiciones de trabajo: tiempo de las jornadas, monto del pago, facilidad de la movilidad de los trabajadores, precariedad e inseguridad de las instalaciones, etc.).

"La subcontratación y tercerización de las fases productivas con menor valor agregado facilitan la existencia de condiciones laborales informales en busca de un menor costo por prenda» (Benencia, 2009), situación que es aprovechada por los emprendedores coreanos. De hecho, como se verá a continuación, la primera instancia de la cadena en general es el trabajo a domicilio o en el pequeño taller de confección. En los primeros tiempos los migrantes coreanos eran subcontratados por los comerciantes de origen judío. Luego, ellos contrataban bolivianos y ahora, contratan a talleristas que, a su vez, contratan en general a otros trabajadores para la parte del proceso de producción que utiliza la mayor parte de obra intensiva.

\section{Etapas y características de la instalación de los migrantes coreanos en la industria textil}

\section{Los inicios de un enclave étnico}

Al llegar a la Ciudad de Buenos Aires a fines de los años 1960 y 1970, los migrantes se instalaron en barrios precarios en donde consiguieron los primeros trabajos de tejido por encargo. Realizaban el trabajo a domicilio, en un primer momento encargados por los comerciantes textiles de Once, casi en su totalidad de la colectividad judía. Este tipo de trabajo (tejidos, pegado de botones, costura y bordado, en la mayoría de los casos) ocupaba a toda la familia durante largos días de labor. Los miembros de la familia no recibían un salario y tampoco se pagaban impuestos por ser declarado trabajo de unidad familiar, permitiéndoles acelerar el proceso de acumulación. La clave simbólica de estos primeros momentos es la lealtad y la confianza dentro de la familia.

Este tipo de trabajo articulaba los intereses de dos grupos presentes en una misma estructura económica, a saber, la imperiosa necesidad de trabajar de los migrantes - caracterizada por una situación marginal a causa del desconocimiento del idioma, de los códigos sociales del nuevo escenario, de las calles y barrios de la ciudad, de su actividad económica - y la necesidad del grupo de comerciantes textiles de Once de contar con mano de obra barata, rápida y eficiente, y que no requería de ningún tipo de mantenimiento.

El trabajo intensivo de todos los miembros del grupo permitía la acumulación de cierto capital con el cual se compraban máquinas y mejoraban la calidad y cantidad del trabajo. Una vez establecidos con máquinas y lugar de trabajo fijo, comienzan a fabricar sus propios productos que venden directamente a los comercios. El crecimiento en este campo se vio favorecido por el hecho de 
que contaban incondicionalmente con la mano de obra familiar y una rígida conducta disciplinada. Esto comenzó a extenderse al punto de que en muy poco tiempo se crearon en esos asentamientos lugares especiales donde daban trabajos de tejido a personas de origen coreano. De esta manera, en 1967 se abre el primer taller de ropa de propietarios coreanos en el barrio de Baek-ku, y en 1973 se abren los primeros locales de venta de ropa de propietarios coreanos en el Barrio de Once. Un año más tarde recién se firmaría el Acta que permitía ingresos familiares de coreanos a la Argentina.

En este momento comienzan a emplear trabajadores, que pueden o no pertenecer a la comunidad. Los migrantes coreanos encontraron puentes de sociabilidad con otros grupos migrantes en la actividad textil, industrial y comercial. Usualmente, los coreanos emplean personal local cuando se trata de negocios con atención al público, pero manteniendo siempre un encargado o supervisor coreano. En cambio, en los talleres textiles, prefieren trabajar con personas provenientes de países limítrofes, bolivianos por lo general. Según el estudio realizado por los antropólogos Bialogorski y Barman (1994), "si los coreanos prefieren tomar trabajadores bolivianos antes que argentinos, esto se debe a una doble consideración: una referida al plano normativo - su situación de residentes ilegales e indocumentados -, la otra, referida al discurso simbólico: en el imaginario de los coreanos, los bolivianos son sumisos y disciplinados».

Por otra parte, contaban con la red solidaria comunitaria, especialmente con el sistema de "crédito rotativo", el $k y e,^{13}$ que permitía invertir esas sumas en los negocios. ${ }^{14}$

Cuando la situación se estabiliza hay quienes abren su propio negocio al público y quienes mantienen el taller como proveedores de otros negocios, y comienzan a subcontratar. De la mano de este proceso de crecimiento y estabilidad económica es que se va delineando la consolidación de esta comunidad.

La actividad textil de coreanos y bolivianos reconoce un antes y un después de la crisis argentina de 2001. Hasta ese año los coreanos dominaban el circuito económico textil (producción y venta con contratación de mano de obra boliviana), aunque durante fines de los años 1990 los migrantes bolivianos comenzaron a dedicarse a la venta de esa producción en las ferias, sobre todo, en aquella que se formó a orillas del Riachuelo en el partido de Lomas de Zamora, conocida como La Salada. Al mismo tiempo, se dio un proceso de re-emigración, como ya se mencionó antes. Luego de la crisis referida, surgió

13 El kye es un tipo de asociación cooperativa que existe en Corea desde la Dinastía Yi. Fue creada para promover asistencia mutual, la amistad y el bienestar. En la Corea actual el kye se modificó para dar lugar a un sistema asociativo de crédito rotativo. En Argentina los residentes coreanos continuaron utilizando este tipo de red asociativa, donde cada miembro ponía una suma, el capital acumulado se sorteaba y se adjudicaba por mes a cada participante. Los primeros migrantes de la década de 1960 y 1970 utilizaron este sistema para invertirlo en los negocios, con sumas que en los 90 llegaron a los 200 mil dólares. Esto funcionó muy bien en los inicios, pero durante la década de 1990 hubo varios fracasos, basados en traiciones de algunos de los miembros que de un día para otro se iban, estafando a todos los integrantes del kye.

14 Nuestras entrevistas en Argentina coinciden con la información de Park Kyeyoung, quien sostiene que "during the 1980 s, Koreans raised business acquisition capital through their own financial institution, the kye (pronounced Keh) rotating credit club» (1997: 58). 
la producción textil entre los bolivianos, repitiendo el ciclo de contrataciones dentro la comunidad misma (bolivianos que producen y venden y contratan mano de obra boliviana). La construcción de espacios de encuentro de diferentes grupos en el mundo del trabajo evidencia tensiones a causa de las relaciones de jerarquía y dominación signadas por la diferencia étnica. (Negocios con dueños/jefes coreanos y empleados bolivianos o peruanos, etc.). A su vez, los coreanos tienen relaciones laborales de tipo de complementariedad con otros coreanos en aparentes igualdades: proveedores, clientes, aprendices. A partir del 2011, comienza a existir competencia entre los coreanos y bolivianos ya que estos últimos han progresado en el comercio textil y se benefician por ciertas situaciones de informalidad que los coreanos ya han formalizado.

La concentración migrante en el mundo textil se debe a una dinámica propia del proceso de establecimiento en el ámbito del trabajo, basado en una red étnica solidaria y en rasgos laborales fuertemente disciplinados que se condicen adecuadamente a las características de la producción textil, que requiere de mano de obra flexible, intensiva, sin capacidad o competencias específicas. Esto se complementa con la propuesta de Wilson y Portes (1980: 295-319), como lo menciona Kyeyoung Park, en su estudio sobre los coreanos en USA:

«Enclaves are places where a group's culture, identity and internal solidarity are preserved. For such an enclave to emerge there must be substantial numbers of immigrants, sufficient sources of capital, and an available suplie of ethnic labor. Portes and Bach cite Koreans of Los Angeles and Cubans of Miami as examples of establish ethnic enclaves. In such enclaves, newly arrived immigrants can survive with little knowledge of the hots culture and language» (Park, 1997: 58).

Además, las actividades económicas que realiza la comunidad sirven tanto al mercado en general (extra-comunitario) como al mercado étnico (intra-comunitario). Cuando argentinos y coreanos se refieren a este punto, coinciden en afirmar la integración de estos últimos en la economía nacional al reconocer el desarrollo de esta actividad en cuya implementación los fabricantes coreanos introdujeron modificaciones concretas en el mercado local que los beneficiaron, al tiempo que beneficiaron a los consumidores, activaron un rubro relegado por la falta de inversiones y en situación crítica desde los años 1980 y contribuyeron al desarrollo de dos zonas comerciales de la ciudad de Buenos Aires (los barrios de Flores y Once), en las que tradicionalmente se asentaban comerciantes y empresarios textiles argentinos de origen judío y armenio (Bialagorski, 2005).

Por otra parte, la concentración de la mayoría de los migrantes coreanos en una determinada rama de la actividad económica (textil) puede ser explicada a partir de la imposibilidad de continuar con sus antiguas profesiones por falta de equivalencias de diplomas, a causa del mal manejo de la lengua, la ausencia de contactos sociales necesarios para emprender trabajos más especializados o el desconocimiento de la sociedad receptora, entre otros. La consolidación paulatina de la red étnica en el rubro textil funcionó como un imán a aquellos que no encontraban otras formas de inserción. 


\section{Concentración de las actividades económicas en Once, Avellaneda, y servicios «étnicos» en Baek-ku, Bajo Flores}

\section{Once}

Barrio central de la ciudad que cuenta con diversos medios de transporte: subterráneos, colectivos, estación de tren y de ómnibus. Se trata de una zona comercial de ventas al por mayor y menor. Hasta la llegada de los coreanos era visto como el barrio de los comerciantes judíos, quienes en gran parte se han desplazado hacia otras zonas. En el discurso cotidiano, los vecinos consideran a los coreanos como los competidores de los judíos y los ven como responsables de su partida. Pero, de acuerdo a relatos de representantes de la comunidad judía, los judíos continúan dominando gran parte del capital económico de Once y son ellos quienes han ofrecido las primeras actividades y trabajos en la rama textil a los coreanos, posibilitando así la entrada de esta comunidad en el sistema económico del barrio y de la ciudad.

El barrio de Once se caracteriza por ser un lugar de aprovisionamiento de mercadería barata. Los locales del barrio poseen una fisonomía en común: marquesinas de colores, carteles en grandes dimensiones, vidrieras sobrecargadas con mercaderías, maniquíes y diversos objetos de adorno, que muchas veces no tienen nada que ver con el negocio y los productos ofrecidos al público. En general las cajas de los negocios son atendidas por los dueños: coreanos o judíos (en la mayoría de los casos, los integrantes de ambas colectividades poseen características exteriores que marcan el estilo de su particularismo cultural, en un caso el ser practicantes de una religión que exige ciertos signos visibles, en el otro por la singularidad de sus rostros), mientras que la atención al público en los negocios coreanos está en manos de empleados no coreanos. Con el apogeo de la comunidad en la década de 1990, se creó una Asociación Coreana de Comerciantes de Once cuyos objetivos eran, por un lado, poner a disposición de estos comerciantes las leyes laborales y del comercio, y por otro, dar conocimiento de las normas de convivencia urbana.

A partir de 1997, debido a la crisis económica que empieza a golpear a los pequeños comerciantes, muchos negocios de propietarios coreanos de Once cierran o se desplazan hacia la Avenida Avellaneda, que comienza a tener mayor relevancia para la comunidad.

\section{Baek-ku, Bajo Flores - Parque Chacabuco}

La experiencia de caminar por el Bajo Flores enfrenta al caminante con una vivencia intercultural en el seno de un antiguo barrio porteño. Predomina en el espacio urbano la presencia de un sistema cultural particular, que se impone sobre la fisonomía de la ciudad. Es un barrio periférico con respecto al centro de la ciudad y a los centros comerciales de otros barrios que lo rodean. Sin embargo, es una zona con una óptima red de trasporte público: colectivos hacia todos los puntos importantes de la ciudad como la Estación de Retiro, Once y Constitución, línea de subterráneo y grandes avenidas que conectan al barrio con el resto de la ciudad. También es destacable la presencia de grandes veredas que durante el auge de la comunidad permitieron una vida social importante. En ese período era muy común ver las calles y las veredas del barrio regadas de grupos de todas las edades que se desplazaban entre iglesias, comercios y 


\section{Figura 2: Instalación en la Ciudad}

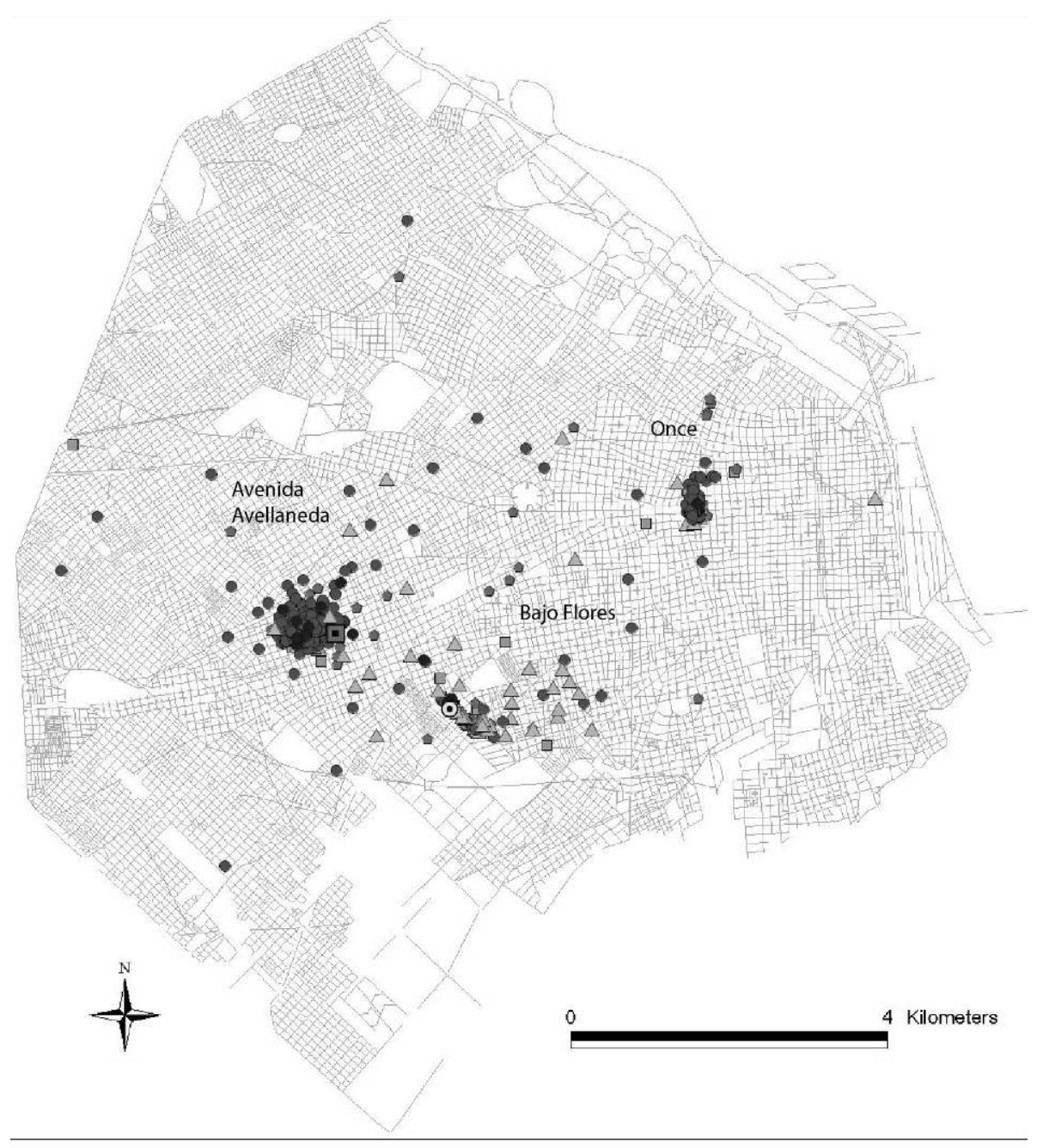

\section{RUBROS}

- Textil

Alimentación

- Salud

$\triangle$ Iglesias

- Educación $\triangle$ Otros comercios

- Colegio Coreano (ICA)

■ Asociación de Comerciantes y Empresarios

Fuente: Brenda Matossian. Realizada con datos del anuario (Han in lok) del 2005. 
servicios, haciendo uso de ese espacio público como parte de sus hábitos cotidianos. Esta característica comienza a desaparecer después de la crisis del 2001, con la disminución numérica de vecinos coreanos en el país, pero sobre todo con el aumento de la inseguridad.

Podemos rastrear hacia finales de 1960 el comienzo de la instalación de Baek-ku. Está situado en el límite entre la zona oeste de la capital y la provincia de Buenos Aires. El barrio está habitado por sectores medios, al límite de una villa de emergencia donde residen otros migrantes internos y de países limítrofes.

Como se observa en la figura 2, los coreanos se han instalado principalmente en una avenida central del barrio de Flores: la Avenida Carabobo. Comienza en Avenida Rivadavia y a unas 15 cuadras de ésta cambia su nombre, a Corea o calle Corea ${ }^{15}$ en reconocimiento a la oleada inmigratoria que desde 1960 contribuyó al crecimiento del barrio. Se encuentran allí todo tipo de comercios, la mayoría de los cuales tenían hasta finales de la década de 1990 los carteles y las leyendas escritas en coreano: panaderías con productos típicos, videoclubes donde incluso los videocasetes de películas occidentales llevan el título en el idioma de los comerciantes, casas de modas, salones de belleza, peluquerías, dentistas, garajes, vidrierías, talleres mecánicos, supermercados con productos con embalajes coreanos, casas de arroz, restaurantes ubicados al final de largos pasillos, pescaderías, agencias inmobiliarias, casas de computación, casa de regalos, una librería y una biblioteca con sistema de alquiler de libros, casas de fotografía, el Golf Shop, la Confederación Argentina de TAE KWON DO, estudios contables, una farmacia, compañías de remises, agencias de viaje y turismo y casas de computación, entre otros negocios.

Se trata de comercios que se dirigen a población coreana, y con la clara evidencia de dejar de lado al resto de la población a través del uso de la escritura en coreano. ${ }^{16}$ No desarrollan ninguna estrategia para llegar al público no coreano. En la mayoría de los casos, los vendedores no hablan español, los productos no están preparados para la venta y consumo de no coreanos, sea porque no ingresan dentro de las típicas comidas argentinas (pescado seco, algas de mar, nabos gigantes y otras verduras aún no conocidas en Argentina) o simplemente porque a causa del tipo de embalaje resulta casi imposible identificar el contenido.

La decoración exterior de los negocios Ilama la atención ya que tanto los vidrios como las vidrieras, por lo general, están pintados con colores opacos o polarizados y no dejan ver el interior. En muchos casos resulta imposible reconocer el tipo de actividad que en ellos se realiza.

En el barrio crecieron espacios que permiten desarrollar una vida asociativa intensa. Algunos ejemplos son: 1) bares y cantobares para las diferentes edades; 2 ) iglesias, encargadas de hacer la conexión entre los individuos de los diferentes espacios de la comunidad; se encuentran allí la iglesia católica, la mayoría de las

15 La Avenida pasa a llamarse de esta manera a partir del año 1989 por adjudicación oficial del Gobierno de la Ciudad de Buenos Aires.

16 Hasta aproximadamente el año 1995 la mayoría de los carteles estaban escritos en coreano, pero por reclamos de los vecinos esta situación se ha modificado hacia una convivencia de ambas lenguas. 
iglesias evangelistas de la comunidad coreana registradas en Buenos Aires y los dos templos budistas; 3 ) asociaciones, medios de comunicación (diarios y radio) y comercios varios. Además de la Asociación Coreana en Argentina existen otras de carácter muy diverso: por origen de provincia, por universidades y escuelas de graduación en Corea, por actividades laborales desarrolladas en Corea antes de emigrar, deportivas, artísticas y de escritores. Hay, asimismo, asociaciones relacionadas con las diversas opciones de vida en la ciudad de Buenos Aires: de comerciantes, de industriales, de profesionales (médicos, abogados, contadores, etc.) de estudiantes, de jugadores de golf y de ancianos. Entre las mismas también se encuentran el Club de calígrafos, el Grupo de estudio de la cultura de los inmigrantes coreanos, el Grupo de Bellas Artes y las iglesias cristianas. Esta amplia gama de instituciones juega un rol muy importante en la forma en que las personas ordenan su vida cotidiana y contribuye fuertemente al desarrollo de la vida de la comunidad (Mera, Palacios de Cosiansi et González, 2005: 59-84). El corazón de la vida asociativa en el barrio de Bajo Flores-Parque Chacabuco experimenta un proceso de crecimiento cualitativo acelerado, se consolidan las asociaciones existentes y surgen nuevas como la Asociación de Profesionales Universitarios Coreanos (APUC), Asociación de Universitarios Coreanos en Argentina (AUCA), Medicina Integral Coreana (MIK), Instituto Coreano Argentino (ICA), nuevos periódicos, Centro de Información de la Cultura Coreana en Argentina, Asociación de escritores, de pintores, etc.

Más allá de la zona centro se instalan las viviendas particulares y algunos otros servicios de manera dispersa por las calles del barrio. Sin embargo, como ya se puntualizó, el impacto de la crisis del 2001 y el aumento de la inseguridad, desplazó muchos negocios de servicios hacia la zona comercial de la Avenida Avellaneda. De todas maneras, dado que las principales iglesias, la escuela (ICA) y otros servicios persisten en esta zona, el barrio continúa con su funcionalidad. Se constata un empobrecimiento y decaimiento general del barrio de Baek-ku en la actualidad. Esto se visualiza en la disminución de comercios y locales de servicio, oscurecimiento de las calles del barrio y deterioro del espacio público como plazas, calles y veredas. La inseguridad es un factor primordial de esquivamiento de la zona en la vida cotidiana. Los negocios que tenían sus instalaciones abiertas o aquellos que tenían productos en la vereda se han también trasladado, en su mayoría, a la concentración en la Av. Avellaneda.

\section{Av. Avellaneda}

Por un lado, la crisis económica repercutió en la situación de muchos comerciantes que eran propietarios de más de un negocio en el Barrio de Once y en la Av. Avellaneda. Ante la imposibilidad de mantener todos los negocios se vieron obligados a optar por uno de ellos. En la mayoría de los casos privilegiaron el negocio de la Av. Avellaneda. Por esta razón, se desarrolla un doble desplazamiento: por un lado, un corrimiento importante de los comercios de Once hacia la zona de la Avenida Avellaneda; por otro, a causa de la cada vez mayor inseguridad en el Barrio de Baek-ku, muchos comerciantes comenzaron a trasladarse hacia la Av. Avellaneda y calles colindantes. Al desplazamiento de las actividades comerciales le siguieron los restaurantes y otros servicios vinculados a la mayor actividad cotidiana, haciendo de esta zona el Centro de mayor importancia económica de la comunidad. En la actualidad allí se concentra la parte más significativa de la actividad textil y de confección, y cada vez más servicios étnicos. 


\section{A modo de reflexión}

El artículo explora el desempeño de la comunidad coreana de Buenos Aires en el mercado textil de la ciudad. Prima la tendencia a generar actividades de micro-emprendimientos independientes y caracterizados por emplear mano de obra intensiva de origen migrante en condiciones muy precarias. El tipo de inserción en la economía local, fundamentalmente su concentración en la pequeña y mediana industria textil es abordada desde la forma de inscripción en el espacio; desde las relaciones sociales inter e intra étnicas en el ámbito del rubro textil - especialmente el diálogo con otros actores no coreanos como grupos de la comunidad judía y migrantes limítrofes, en su mayoría de Bolivia -; y desde las posibilidades y el sentido simbólico que estos comportamientos adquieren para los miembros de esta comunidad.

Las dinámicas de la economía étnica dan sentido a la forma en que estos grupos se instalan y organizan: los roles que juegan la familia, las iglesias y las asociaciones estructuran la vida comunitaria; el patrón concentrado de instalación en el espacio urbano fomenta las relaciones intra-etnia; el desenvolvimiento "exitoso" en el ámbito económico local se explica a partir de la consolidación del modelo del empresariados étnicos; y la construcción de una red étnica confiere posibilidades de reforzar los mecanismos de transmisión y creación de valores propios.

El capital social y cultural que provee la comunidad se plasma también en los recursos materiales que facilitan la concentración en determinadas zonas de la Ciudad y en rubros delimitados de la economía.

Respecto a las actividades económicas, se basan en una gran dependencia de las redes étnicas. En la aparición de empresariados étnicos influyen las estrategias de los grupos migrantes pero también las condiciones del contexto de recepción, marcado por la recesión económica y la utilización de la mano de obra indocumentada. Estos empresariados se caracterizan por desarrollar actividades fundamentalmente entre personas también provenientes de Corea, y con una significativa, aunque no absoluta, dependencia del capital social proporcionado por los recursos de las propias redes étnicas (empleo e intercambio entre co-étnicos, ayuda familiar, facilidades de préstamo de dinero entre parientes, amigos y vecinos). Asimismo, se puede concluir que la organización de redes étnicas posibilita ciertas tendencias a la consolidación de relaciones endogámicas en la actividad económica pero que esto no garantiza su éxito económico. Se confirma que es la capacidad adaptativa, de uso y de apropiación del espacio (en sus formas de concentración, dispersión, circulación), lo que complementariamente explica ese relativo éxito. Los migrantes coreanos logran establecerse como emprendedores independientes y conservar una fuerte identidad propia gracias a las estrategias asociadas a la concentración en el espacio urbano y en la economía de servicios étnicos que provee empleo y beneficios a los grupos coreanos frente a una sociedad que, en un principio, les es impropia.

La diferencia en cuanto a la instalación producto del rubro económico dominante marca la relación con los no comunitarios, y la forma en que se articulan las relaciones étnicas. Es decir, la forma de instalación, apropiación y circulación del/por el espacio urbano promueve, entre la población local, dife- 
rentes representaciones de estos migrantes, ya que implica diferentes formas de relaciones con los extracomunitarios. La relación con los migrantes coreanos y su concentración espacial, económica y residencial ha generado en muchos casos ciertos discursos discriminadores y representaciones negativas del grupo.

El desarrollo de las economías étnicas facilita a los migrantes el poder hacer frente a la inserción en los nuevos escenarios de recepción y circulación, muchas veces signados por economías frágiles, políticas inestables y discursos discriminadores, con los cuales estos migrantes deben aprender a relacionarse.

\section{Bibliografía}

Arfuch Leonor (2005) Pensar este tiempo. Espacios afectos, pertenencias, Buenos Aires, Paidós, 332 p.

Barth Frederik (1976) Los grupos étnicos y sus fronteras. La organización social de las diferencias culturales, México, Fondo de Cultura Económica, 204 p.

Bauman Zygmunt (1999) La globalización. Consecuencias humanas, Buenos Aires, FCE, $171 \mathrm{p}$.

Benencia Roberto (2009) El infierno del trabajo esclavo. La contracara de las 'exitosas' economías étnicas, [en línea] consultado el 13/09/2012. URL: http://www. scielo.org.ar/scielo.php?script=sci_arttext\&pid=S1851-16942009000200002

Bialogorski Mirta (2005) La experiencia coreana en Argentina: ¿hacia una construcción de la integración?, II Encuentro Latinoamericano de Estudios Coreanos, [en línea] consultado el 14/09/2012. URL: http://ceaa.colmex.mx/estudioscoreanos/images/mirta.pdf

Bialogorski Mirta y Barman Daniel (1994) La mirada del otro: coreanos y bolivianos en Buenos Aires, Seminario sobre Discriminación y Prejuicio en Latinoamérica, Buenos Aires, Instituto de Investigaciones en Historia Social y económica, Facultad de Ciencias Económicas, UBA.

Bonacich Edna (1973) A theory of middleman minorities, American Sociological Review, 38 (5), pp. 583-594.

Cohen Néstor y Mera Carolina (Comps) (2005) Relaciones interculturales: experiencias y representación social de los migrantes, Buenos Aires, Antropofagia, $222 \mathrm{p}$.

D’Ovidio María (2007) ¿Quién es quién en la cadena de valor del sector de indumentaria textil? Hacia una solución conjunta en el sector, [en línea] consultado el 14/09/2012. URL: http://www.inti.gov.ar/sabercomo/sc53/inti7.php

Grafmeyer Yves et Joseph Isaac (1990) [1932] L'école de Chicago. Naissance de l'écologie urbaine, Paris, Aubier, $377 \mathrm{p}$.

Hurh Won Moo (1980) Toward a new community and identity: the koreanamerican ethnicity, in Kim Byong-Suh and Lee Sang Hyun Eds., The Korean Immigrants in America, Montclair, The Association of Korean Christian Scholars in North America, pp. 1-36.

Kim Kwang Chung et Hurh Won Moo (1993) Beyond assimilation and pluralism: syncretic sociocultural adaptation of korean inmigrants in the USA, Ethnic and Racial Studies, 16 (4), pp. 696-713. 
Kim Kwang Chung et Hurh Won Moo (1984) Adhesive Sociocultural Adaptation of Korean Immigrants in the U.S.: An Alternative Strategy of Minority Adapation, International Migration Review, 18 (2), pp. 188-216.

Kim Kwang Chung, Kim Hei Chu y Hurh Won Moon (1981) Job information deprivation in the United Satates: A case study of Korean immigrants, Ethnicity, 8 (3), pp. 219-232.

Lee Kyo Bom (1990) La historia de la migración coreana en Argentina, Buenos Aires, Sonyoungsa, $301 \mathrm{p}$.

Light Ivan (1972) Ethnic enterprise in America, Berkley, University of California Press, $209 \mathrm{p}$.

Mármora Lelio (1971) Modelo analítico del proceso de inserción migratoria, Aportes, 21.

Martin Saravia Rodolfo (1988) La emigración coreana en la República Argentina, in Liliana García Daris Comp., Corea. Antigüedad y actualidad, Buenos Aires, Eudeba, pp. 179-189.

Massey Doreen (2005) La filosofía y la política de la espacialidad: algunas consideraciones, in Leonor Arfuch Comp., Pensar este tiempo. Espacios afectos, pertenencias, Buenos Aires, Paidós, pp. 103-127.

Mera Carolina (2011) Movilidad territorial en la Ciudad de Buenos Aires. Sobre los patrones residenciales de las migraciones chinas y coreanas, in Ariel Guiance Comp., Movilidad y Migraciones, Buenos Aires, CONICET-IMHICIHU, pp. 201-212.

Mera Carolina (2010) El concepto de diáspora en los estudios migratorios: reflexiones sobre el caso de las comunidades y movilidades coreanas en el mundo actual, Revista de Historia, 12, pp. 1-18.

Mera Carolina (2008) La comunidad coreana en Buenos Aires. Una experiencia de convivencia intercultural, Revista Sociedad, 27, pp. 1-10.

Mera Carolina (1998) La inmigración coreana en Buenos Aires. Multiculturalismo en el espacio urbano, Buenos Aires, Eudeba, 182 p.

Mera Carolina, Palacios de Cosiansi Liliana y González Carmen (2005) Modalidad de instalación de la comunidad coreana en la Ciudad de Buenos Aires, in Carolina Mera, Liliana Palacios de Cosiansi et Carmen González Comps., Coreanos en Argentina: 40 años de Historia, La Plata, Al Margen, pp. 59-84.

Min Pyong Gap (1990) Problems of Korean Immigrants Entrepreneurs, International Migration Review, 24 (3), pp. 436-455.

Min Pyong Gap and Kim Rosa (Eds.) (1999) Struggle for ethnic identity: narratives by Asian American professionals, Walnut Creek, Altamira Press, 240 p.

Park Kyeyoung (1997) The Korean American Dream: Inmigrants and Small Business in New York City, Ithaca, Cornell University Press, 228 p.

Sassen Saskia (1999) La ciudad global, Nueva York, Londres, Tokio, Buenos Aires, Eudeba, $458 \mathrm{p}$.

Sassone Susana (2004). Identidad Cultural y Territorio: La construcción del "lugar» en la comunidad de migrantes bolivianos en la Zona Sur de la Ciudad de Buenos Aires, in Andrzej Dembicz Ed., Interculturalidad en América Latina en ámbitos locales y regionales, Warsawa, Universidad de Varsovia, CESLA, pp. 177-197. 
Sassone Susana (2002) Geografías de la exclusión. Inmigración limítrofe indocumentada en la Argentina: Del Sistema-Mundo al Lugar. Tesis Doctoral, Mendoza, Universidad Nacional de Cuyo, Facultad de Filosofía y Letras.

Tarrius Alain (2000) Leer, describir, interpretar. Las circulaciones migratorias: Conveniencia de la noción de "Territorio circulatorio". Los nuevos hábitos de la identidad, Revista Relaciones. Estudios de Historia y Sociedad, 21 (83), pp. 37-66.

Waldinger Roger, Aldrich Howard and Ward Robin (1990) Ethnic entrepreneurs. Immigrants business in industrial society, London, Sage Publications, 226 p.

Wilson Kenneth and Portes Alejandro (1980) Immigrant Enclaves: An Analysis of the Labor Market Experiences of Cubans in Miami, American Journal of Sociology, 86 (2), pp. 295-319.

Yoon In-Jin (1991) The changing significance of ethnic and clases resources in immigrant business: the case of Korean Immigration, International Migration Review, 25 (2), pp. 303-332. 


\section{Carolina Mera}

\section{Los migrantes coreanos en la industria textil de la Ciudad de Buenos Aires. Inserción económica e identidades urbanas}

El artículo explora el desempeño de la comunidad coreana de Buenos Aires en el mercado textil de la ciudad. Se trata de comprender los mecanismos que interactúan en esta área de la industria que impacta en la forma de organización social del grupo, teniendo en cuenta las dinámicas (momentos de crisis y mejoras) de la economía local-global. En este marco presentaremos en primer lugar una caracterización del proceso de llegada e instalación de esta comunidad en Argentina, su naturaleza diaspórica y la importancia del capital social. Se delinean los aspectos más generales de la comunidad coreana en Buenos Aires: el rol que juegan la familia, las iglesias y las asociaciones en la forma de estructurar la vida comunitaria; el patrón concentrado de instalación en el espacio urbano; el desenvolvimiento de manera «exitosa» en el ámbito económico local; y la construcción de una red étnica posible de reforzar los mecanismos de transmisión y creación de valores propios. Luego, indagaremos en el tipo de inserción en la economía local, fundamentalmente su concentración en la pequeña y mediana industria textil. Abordaremos este eje en tres aspectos: la forma de inscripción en el espacio urbano; las relaciones sociales inter e intra étnicas en el ámbito del rubro textil; y las posibilidades y el sentido que estos comportamientos adquieren para los miembros de esta comunidad en el ámbito local-global. Finalmente, las conclusiones se presentan como aportes para pensar el mundo contemporáneo desde la experiencia de estas comunidades migrantes diaspóricas en un mundo cada vez más transnacional.

\section{Les migrants coréens dans l'industrie textile de la ville de Buenos Aires. Intégration économique et identités urbaines}

L'article explore le rôle de la communauté coréenne de Buenos Aires dans le marché textile de la ville. Il s'agit à la fois de comprendre les mécanismes qui interagissent sur le terrain de l'industrie et leur influence sur les formes de l'organisation sociale du groupe en regard des dynamiques (moment de crise et d'amélioration) de l'économie locale-globale. Dans ce cadre, l'article présente dans un premier temps une caractérisation du processus d'arrivée et d'installation de cette communauté en Argentine en y explorant les mécanismes de transmission et de création de valeurs propres au groupe ainsi que le rôle des réseaux familiaux et plus largement communautaires dans leur " réussite " économique au niveau local. Cette dernière question fait l'objet d'un second développement où est analysé le modèle d'insertion dans l'économie locale au prisme de trois approches : les formes d'ancrage dans l'espace urbain ; les particularités des relations sociales inter et intra ethniques développées dans le secteur de l'industrie textile; et les possibilités et le sens que ces comportements offrent aux membres de cette communauté dans le cadre local-global. Au final, les conclusions se présentent comme des données permettant de penser le monde contemporain à partir de l'expérience de communautés migrantes diasporiques inscrites dans un monde de plus en plus transnational. 


\section{Korean Migrants in the Buenos Aires Garment Industry. Economic Integration and Urban Identities}

This article explores the participation of the Buenos Aires Korean community in the city's garment market. It aims at understanding the mechanisms that interact in this sector to influence the group's social organization, taking into account the dynamics (times of crisis and of upturns) in the local-global economy. First, within this context, I offer a description of this community's arrival and settlement in Argentina, its diasporic nature and the importance of social capital. I outline the major general aspects of the Korean community in Buenos Aires: The role played by the family, churches and associations in how community life is structured; the concentrated settlement pattern in the urban space; the community's "successful" development in the local economic sphere. Second, I investigate the community's form of integration in the local economy - primarily its concentration in the small and medium garment industry. I address this in three aspects: The form of inscription in the urban space; inter- and intra-ethnic social relations within the garment sector; and the possibilities and the meaning that these conducts take on for the members of this community in the localglobal sphere. Finally, I present conclusions as contributions for thinking about the contemporary world based on the experience of these diasporic migrant communities in an increasingly transnational world. 